\title{
Detection of ultra-high-energy neutrinos by IceCube: sterile neutrino scenario
}

\author{
Subhash Rajpoot ${ }^{1, \mathrm{a}}$, Sarira Sahu ${ }^{2, \mathrm{~b}}$, Hsi Ching Wang ${ }^{1, \mathrm{c}}$ \\ ${ }^{1}$ Department of Physics and Astronomy, California State University, 1250 Bellflower Boulevard, Long Beach, CA 90840, USA \\ ${ }^{2}$ Instituto de Ciencias Nucleares, Universidad Nacional Autónoma de México, Circuito Exterior, C.U., \\ A. Postal 70-543, 04510 Mexico, DF, Mexico
}

Received: 20 January 2014 / Accepted: 6 June 2014 / Published online: 26 June 2014

(C) The Author(s) 2014. This article is published with open access at Springerlink.com

\begin{abstract}
The short-baseline neutrino oscillation experiments, the excess of radiation from the measurement of the cosmic microwave background radiation, the necessity of the nonbaryonic dark matter candidate, and the depletion of the neutrino flux in IceCube all seem to hint at new physics beyond the standard model. An economical way to address these issues is to invoke the existence of sterile neutrinos. We present simple extensions of the standard model with additionally three sterile neutrinos and discuss the corresponding PMNS like neutrino flavor mixing matrix. The noteworthy features of the sterile neutrino scenario advocated here are that the lightest one is almost degenerate with one of the active neutrinos, the second sterile has mass of order $\mathrm{eV}$, and the heaviest one is in the $\mathrm{keV}$ range. In the present scenario, the short-baseline anomaly is explained through $\Delta m^{2} \sim 1 \mathrm{eV}^{2}$, the depletion of the muon neutrino flux in IceCube is explained through $\Delta m^{2} \sim 4.0 \times 10^{-16} \mathrm{eV}^{2}$, and the dark matter problem is addressed through $\Delta m^{2} \sim 1 \mathrm{keV}^{2}$. Our proposed mixing matrix is also compatible with the observed neutrino oscillation data. We show that the highenergy muon and the tau neutrino fluxes from Gamma Ray Bursts can be depleted in IceCube by as much as 38 and $43 \%$, respectively. These substantial depletions in both muon and tau neutrino fluxes are due to their small but sizable mixing with the sterile neutrinos.
\end{abstract}

\section{Introduction}

In the standard picture of neutrino oscillations, the three active neutrino states are linear superpositions of three mass eigenstates. The oscillation experiments with solar, atmo-

\footnotetext{
a e-mail: Subhash.Rajpoot@csulb.edu

be-mail: sarira@nucleares.unam.mx

c e-mail: Hsi-ching.Wang@cgu.edu
}

spheric, reactor, and accelerator neutrinos can be explained through the mass squared differences $[1,2]$

$$
\begin{aligned}
& \Delta m_{\mathrm{sol}}^{2} \equiv \Delta m_{21}^{2} \simeq 7.6 \times 10^{-5} \mathrm{eV}^{2}, \\
& \Delta m_{\mathrm{atm}}^{2} \equiv \Delta m_{31}^{2} \simeq 2.45 \times 10^{-3} \mathrm{eV}^{2},
\end{aligned}
$$

with $\Delta m_{i j}^{2}=m_{i}^{2}-m_{j}^{2}$. The three mixing angles in this scheme have also been measured. The solar [3] and KamLand [4] data give $\sin ^{2} \theta_{12} \simeq 0.3$; the atmospheric [5] and MINOS [6] data give $\sin ^{2} \theta_{23} \simeq 0.5$. Also recently the DoubleCHOOZ [7], RENO [8] and Daya-Bay [9] experiments measured the third mixing angle, $\sin ^{2} \theta_{13} \simeq 0.1$. However, the completeness of the three-neutrino mixing paradigm is in question due to several anomalies observed in the appearance and disappearance of neutrinos in data pertaining to shortbaseline (SBL) experiments; Liquid Scintillator Neutrino Detector (LSND) [10], Mini-Booster Neutrino Experiment (MiniBooNE) [11], and the reactor anomaly [12] (henceforth all combined and referred to as the SBL anomaly). The SBL anomaly cannot be accommodated with just three active neutrinos, thus suggesting the possible existence of one or more $\mathrm{eV}$-scale sterile neutrinos to explain these results [13].

Although the existence of dark matter (DM) in the Universe is confirmed beyond doubt, its nature is still an outstanding puzzle both in particle physics and cosmology. To be consistent with the observations, the DM candidate should be a very weakly interacting, electrically neutral particle. Sterile neutrinos with a mass $\mathcal{O}(1) \mathrm{keV}$ and lifetimes much longer than the age of the Universe are very good candidates for warm dark matter (WDM) $[14,15]$. These sterile neutrinos could be produced in the early Universe and their mass is generated by a Majorana mass term which is not bound to the electroweak scale. Apart from explaining the DM problem sterile neutrinos may also explain the large pulsar kick velocity [16], and their presence may also suppress the formation of dwarf-galaxies and other small-scale structures. 
Gamma-ray bursts (GRBs) and active galactic nuclei (AGN) are believed to be the prime candidates for the production of ultra-high-energy cosmic rays (UHECRs) and ultra-high-energy neutrinos are their by-products [17-20]. IceCube, the $\mathrm{km}^{3}$-scale neutrino telescope constructed at the South Pole is meant to detect these cosmological neutrinos [21]. The IceCube collaboration recently published their analysis of data taken during the construction phase using the 40- and 59-string configurations of the detector. The combined analysis of the data does not show any neutrino signal correlated with the observed GRBs during the data taking period [22,23]. From this analysis, IceCube has set an upper bound on the neutrino flux from GRBs, which is at least a factor of 3.7 below the Waxman-Bahcall (WB) predication [24]. This depletion in the neutrino flux gave rise to many possible explanations [25-27].

From the astrophysics point of view, it has been pointed out recently that for the normalization of the neutrino flux, IceCube ignored the effects of the energy dependence of the charged pion production and secondary pion/muon cooling in the GRB fireball, which caused an overestimation of neutrino flux by a factor of 5 for typical GRB parameters [28]. Furthermore, by taking into account many other effects (pion and kaon production models, magnetic field effect, and neutrino flavor mixing) and doing a full numerical calculation it is shown that the neutrino flux reduces by about one order of magnitude [29]. With the revised neutrino flux calculation, a reduction in flux is also obtained by analyzing the neutrino flux from 215 GRBs during the period of the 40and 59-string configuration of the IceCube [30]. There are also alternative astrophysical models [31-33], which predict a lower neutrino flux compared to the WB models. So the claim by IceCube may not be that serious, but the WB models can be challenged in the future, as the observations put stringent limits on the muon neutrino flux.

To address this issue from the particle physics point of view, the existence of pseudo-Dirac neutrinos [34-40] is postulated. In this scenario the neutrino of each generation is composed of an almost maximally mixed active-sterile neutrino combination, separated by a tiny mass difference so that the active-sterile oscillations are possible without affecting the short-baseline oscillation results [41,42]. In a recent paper it has been postulated that, apart from the above explanation, neutrino decay can also be a viable explanation for the suppression of the muon neutrino flux [43]. Yet another very recent paper discussed the suppression of the muon neutrino flux in IceCube by assuming that all the neutrinos are pseudo-Dirac in nature and there is a mirror world replicating the interactions in the observed world and also connected to the latter gravitationally. In this scenario each active neutrino is associated with three sterile neutrinos with a very tiny splitting and oscillation from active to sterile can be responsible for the suppression of the muon neutrino flux [44]. So, if sterile neutrinos exist at all, and one/some of them are closely degenerate in mass with the active neutrinos, and also mix, they may easily evade detection in oscillation experiments. However, due to the very long baseline involved in the oscillation process the sterile neutrino, in principle, can have measurable effects on the high-energy neutrino flux. Also the possibility of a sterile neutrino was looked for in the atmospheric neutrino data collected by AMANDA and partially deployed IceCube [45].

These postulated sterile neutrinos neither participate in the weak interaction nor contribute to the invisible width of the $\mathrm{Z}$ boson [13]. Also there is no known fundamental symmetry in nature forcing the existence of a fixed number of sterile neutrino species. Cosmological probes such as bounds on the relativistic energy density of the universe in terms of the effective number of light neutrinos [46] have been extensively used to set bounds on the number of light neutrinos in general and the number of sterile neutrinos in particular.

In this work, we extend the Standard Model to include three additional sterile neutrinos $(3+3)$. All neutrinos in the model, active and sterile, have non-zero masses and mix. The flavor mixing among the neutral leptons gives rise to a $6 \times 6$ matrix, analogous to the PMNS scheme for the active neutrinos. We will show that the generalized $6 \times 6$ matrix is compatible with the observed active neutrino oscillation data. Although our main focus is to explain the depletion of the muon neutrino flux in IceCube, our model also encompasses solutions to the SBL anomaly and the dark matter problem.

\section{The $(3+3)$ model}

We assume that, apart from three active neutrinos, there are three additional sterile neutrinos. The flavor states of the active neutrinos are defined as $\left|v_{e}\right\rangle,\left|v_{\mu}\right\rangle$, and $\left|v_{\tau}\right\rangle$. The corresponding mass eigenstates are $\left|v_{1}\right\rangle,\left|v_{2}\right\rangle$, and $\left|v_{3}\right\rangle$ with masses $m_{1}, m_{2}$, and $m_{3}$, respectively. The flavor states of the sterile neutrinos are defined as $\left|v_{a}\right\rangle,\left|v_{b}\right\rangle$, and $\left|v_{c}\right\rangle$. The corresponding mass eigenstates are $\left|v_{4}\right\rangle,\left|v_{5}\right\rangle$, and $\left|v_{6}\right\rangle$ with masses $m_{4}$, $m_{5}$, and $m_{6}$, respectively.

In the standard treatment of neutrino oscillations in vacuum, the flavor and the mass eigenstates are defined as $v_{\alpha}$ and $v_{i}$, respectively. The flavor states are superpositions of mass eigenstates with a non-zero mass square difference and are given as

$\left|v_{\alpha}\right\rangle=\sum_{i=1}^{6} U_{\alpha i}^{*}\left|v_{i}\right\rangle$.

The mixing matrix $U$ is the extended Pontecorvo-MakiNakagawa-Saki (PMNS) matrix. The three lowest states, $\left|v_{1}\right\rangle,\left|v_{2}\right\rangle$, and $\left|v_{3}\right\rangle$, with their respective masses $m_{1}, m_{2}$, and $m_{3}$ account for solar and atmospheric neutrino oscillations. 
We assume that the sterile neutrinos are Majorana singlets. They could either be left handed or right handed. Below we shall describe two models with Majorana steriles. The first model in which $\left|v_{a}\right\rangle,\left|v_{b}\right\rangle$, and $\left|v_{c}\right\rangle$ are left handed will be referred to as model A, while the second model in which $\left|v_{a}\right\rangle$, $\left|v_{b}\right\rangle$, and $\left|v_{c}\right\rangle$ are right handed will be referred to as model B.

We present a standard model extension in which the seesaw mechanism is invoked to generate the required spectrum of light sterile neutrinos. The standard model with three generations of quarks and leptons is extended in the leptonic sector to include three right handed neutrinos and three vectorlike neutral leptons [47-50]. In total, our model has 12 neutral leptons, three left handed active neutrinos $v_{L} \equiv\left(\left|v_{e L}\right\rangle,\left|v_{\mu L}\right\rangle\right.$, $\left.\left|v_{\tau L}\right\rangle\right)$, their counter parts, the three right handed inert neutri$\operatorname{nos} v_{R} \equiv\left(\left|v_{e R}\right\rangle,\left|v_{\mu R}\right\rangle,\left|v_{\tau R}\right\rangle\right)$, additionally three left handed and three right handed neutrals $N_{L} \equiv\left(N_{1}, N_{2}, N_{3}\right)_{L}, N_{R} \equiv$ $\left(N_{1}, N_{2}, N_{3}\right)_{R}$. The interaction Lagrangian relevant for the neutrino masses and mixings is symbolically given by

$$
\begin{aligned}
-\mathcal{L}_{Y}= & \bar{L}_{i} Y \Phi v_{R}+\bar{L}_{i} Y^{\prime} \Phi N_{R}+\frac{1}{2} \bar{v}_{R}^{C}(M) v_{R} \\
& +\bar{N}_{L}\left(\Delta^{\prime}\right) v_{R}+\frac{1}{2} \bar{N}_{L}\left(M_{L L}\right) N_{L}^{C}+\bar{N}_{R}\left(m_{R R}\right) v_{R}^{C} \\
& +\bar{N}_{L}\left(\Delta^{\prime \prime}\right) N_{R}+\frac{1}{2} \bar{N}_{R}\left(M_{R R}\right) N_{R}^{C}+\text { H.C. }, \quad(3
\end{aligned}
$$

which gives rise to the following $12 \times 12$ neutrino mass matrix in the basis $\left\{v_{L}, v_{R}^{C}, N_{L}, N_{R}^{C}\right\}$ :

$\mathcal{M}_{v}=\left(\begin{array}{cccc}\mathbf{0} & \Delta & \mathbf{0} & \Delta^{\prime} \\ \Delta^{\top} & M & \Delta^{\prime \top} & m_{R R}^{\top} \\ \mathbf{0} & \Delta^{\prime \prime} & M_{L L} & \Delta_{L R} \\ \Delta^{\prime \top} & m_{R R} & \Delta_{L R}^{\top} & M_{R R}\end{array}\right)$.

All entries in $\mathcal{M}_{v}$ are $3 \times 3$ matrices. $Y$ and $Y^{\prime}$ represent Yukawa couplings, $\Delta=Y\langle\Phi\rangle$ is the Dirac mass matrix for the active neutrinos, $\Delta^{\prime}=Y^{\prime}\langle\Phi\rangle$ is the Dirac mass matrix for the active neutrinos and $N_{R} . M$ is the $(B-L)$-breaking Majorana mass matrix of the right handed neutrinos; $\Delta^{\prime \prime}$ is the Dirac mass matrix for $v_{R}$ and $N_{L} \cdot \Delta_{L R}$ is the Dirac mass matrix for $N_{L}$ and $N_{R}$. The remaining terms in $\mathcal{M}_{v}$ are all Majorana mass matrices. The model has enough parameters to give representative values for masses and flavor mixings to address the short-baseline neutrino oscillation experiments, the excess of radiation from the measurement of the cosmic microwave background radiation, the need for nonbaryonic dark matter, and the depletion of the neutrino flux in IceCube.

Model A: In this model the light mass eigenstates are the active states $v_{L} \equiv\left(\left|v_{e L}\right\rangle,\left|v_{\mu L}\right\rangle,\left|v_{\tau L}\right\rangle\right)$, and the sterile neutrals $N_{L} \equiv\left(N_{1}, N_{2}, N_{3}\right)_{L}$. The lightness of the states is achieved by invoking the seesaw mechanism in two stages. The first stage is between the three active neutrinos and their counter parts, the three right handed inert neutrinos $v_{R} \equiv$ $\left(\left|v_{e R}\right\rangle,\left|v_{\mu R}\right\rangle,\left|v_{\tau}\right\rangle\right)$. The second stage is between $N_{L} \equiv$ $\left(N_{1}, N_{2}, N_{3}\right)_{L}, N_{R} \equiv\left(N_{1}, N_{2}, N_{3}\right)_{R}$. These two stages are achieved by constraining the elements of the sub mass matrices in $\mathcal{M}_{v}$ to satisfy the seesaw conditions,

$$
M, M_{R R} \gg \Delta, \Delta^{\prime}, \Delta^{\prime \prime}, M_{L L}, \Delta_{L R}, m_{R R} \text {. }
$$

The light neutrino masses for the three active states are given by

$M_{v_{L}} \simeq-\Delta M^{-1} \Delta^{\top}$.

The active states mix through the matrix elements of $\epsilon=$ $\Delta M^{-1}$. These mixings are responsible for the observed solar, atmospheric, and reactor neutrino oscillations. Similarly, the masses of the light steriles are given by

$M_{N_{L}} \simeq M_{L L}-\Delta_{L R} M_{R R}^{-1} \Delta_{L R}^{\top}$,

and the states mix via the matrix elements of $\delta=\Delta_{L R} M_{R R}^{-1}$. Further mixings between the three active states and the three sterile states are achieved through the off diagonal matrices $\Delta^{\prime \prime}$. These mixings are considered in addressing the reactor anomaly, the flux depletion at IceCube, and dark matter.

Model B: In this model the light mass eigenstates are the active states $v_{L} \equiv\left(\left|v_{e L}\right\rangle,\left|v_{\mu L}\right\rangle,\left|v_{\tau L}\right\rangle\right)$, and their counter parts, the three right handed inert neutrinos $v_{R} \equiv\left(\left|v_{e R}\right\rangle\right.$, $\left.\left|v_{\mu R}\right\rangle,\left|v_{\tau R}\right\rangle\right)$. In this model also the lightness of the states is achieved by invoking the seesaw mechanism in two stages. The first stage is between the three active neutrinos and $N_{R}$ $\equiv\left(N_{1}, N_{2}, N_{3}\right)_{R}$. The second stage is between $v_{R} \equiv\left(\left|v_{e R}\right\rangle\right.$, $\left.\left|v_{\mu R}\right\rangle,\left|v_{\tau R}\right\rangle\right)$, and $N_{L} \equiv\left(N_{1}, N_{2}, N_{3}\right)_{L}$. These two stages are achieved by constraining the elements of the sub mass matrices in $\mathcal{M}_{v}$ to satisfy the seesaw conditions,

$M_{R R}, M_{L L} \gg M, \Delta, \Delta^{\prime}, \Delta^{\prime \prime}, \Delta_{L R}, m_{R R}$.

The light neutrino masses for the three active states are given by

$M_{v_{L}} \simeq-\Delta^{\prime} M_{R R}^{-1} \Delta^{\prime \top}$.

The active states mix through the matrix elements of $\epsilon^{\prime}=$ $\Delta^{\prime} M_{R R}^{-1}$. These mixing matrix elements are responsible for the observed solar, atmospheric, and reactor neutrino oscillations. Similarly, the masses of the light $v_{R}$ are given by

$M_{\nu_{R}} \simeq M-\Delta^{\prime \prime} M_{L L}^{-1} \Delta^{\prime \prime \top}$,

and the states mix via the matrix elements of $\delta=\Delta^{\prime \prime} M_{L L}^{-1}$. Further mixing between the three active states and the three sterile states is achieved through the off diagonal matrices $\Delta^{\prime \prime}$. These mixings are responsible for addressing the reactor anomaly, the flux depletion at IceCube, and dark matter. This model also offers the possibility of constructing a pseudoDirac particle [34-39] by combining two almost degenerate 
mass eigenstates, one from the active neutrinos and another from their right handed counterparts.

\section{High-energy neutrino oscillation}

In the $(3+3)$ model, the vacuum oscillation probability for the process $v_{\alpha} \rightarrow v_{\beta}$ is given as

$$
\begin{aligned}
P_{\alpha \beta}(L)= & \delta_{\alpha \beta}-4 \sum_{i>j} \Re\left[U_{\alpha i}^{*} U_{\beta i} U_{\alpha j} U_{\beta j}^{*}\right] \sin ^{2}\left(\frac{\Delta m_{i j}^{2} L}{4 E_{v}}\right) \\
& +2 \sum_{i>j} \Im\left[U_{\alpha i}^{*} U_{\beta i} U_{\alpha j} U_{\beta j}^{*}\right] \sin \left(\frac{\Delta m_{i j}^{2} L}{2 E_{v}}\right),
\end{aligned}
$$

where ( $i, j=1$ to 6$)$ and we have 15 different $\Delta m_{i j}^{2}=m_{i}^{2}-$ $m_{j}^{2}$ for non-zero and non-degenerate cases. For given $\Delta m^{2}$, the oscillation probability depends on the neutrino energy $E_{v}$ and the propagation distance (baseline) $L$. Because CP violation in the neutrino sector has not been observed yet, we take all the phases to be zero and this makes the $U$ matrix real and simplifies the oscillation probability to the following form:

$P_{\alpha \beta}(L)=\delta_{\alpha \beta}-4 \sum_{i>j}\left[U_{\alpha i} U_{\beta i} U_{\alpha j} U_{\beta j}\right] \sin ^{2}\left(\frac{\pi L}{L_{\mathrm{osc}}}\right)$,

where $L_{\mathrm{osc}}=4 \pi E_{v} / \Delta m_{i j}^{2}$ is the oscillation length. The maximum flavor conversion in the vacuum can take place when $L=L_{\mathrm{osc}} / 2$. If $L \gg L_{\mathrm{osc}}$, the oscillations are very rapid and the oscillating term averages to $1 / 2$. In this case the oscillation probability depends neither on the neutrino energy $E_{v}$ nor on the distance $L$ from the source. On the other hand, if $L \ll L_{\mathrm{Osc}}$, the baseline is too short for neutrinos to oscillate.

In order to explain the solar and atmospheric neutrino oscillation data we take $\Delta m_{21}^{2}$ and $\Delta m_{31}^{2}$ as given in Eq. (1) and their corresponding mixing angles. To explain the SBL anomaly we adopt the $(3+1)$ model [51]. In the $(3+1)$ scenario, the neutrino masses consist of three active neutrinos with masses $m_{1}, m_{2}$, and $m_{3}$, which accommodate the observed solar and atmospheric oscillations, and a sterile state with mass $m_{j},(j=4$ or 5), separated from the active states by $\Delta m_{j 1}^{2} \sim 1 \mathrm{eV}^{2} \gg \Delta m_{21,31}^{2}$. The small squaredmass differences $\Delta m_{21}^{2}$ and $\Delta m_{31}^{2}$ which are responsible for solar and atmospheric neutrino oscillations, respectively, have negligible effects in SBL oscillations. On the other hand, due to the large $\Delta m_{j 1}^{2}$ and small active-sterile mixing, the effects of the sterile neutrino on the solar neutrino oscillation and conventional atmospheric neutrino oscillation $\left(E_{v} \sim \mathrm{GeV}\right)$ are also negligible. However, the new large mass-squared difference $\Delta m_{j 1}^{2} \sim 1 \mathrm{eV}^{2}$ induces an active- sterile oscillation at short baselines $\sim 30 \mathrm{~m}$ for neutrinos with an energy in the range $20 \mathrm{MeV}<E_{v}<200 \mathrm{MeV}$, which is invoked to interpret the SBL anomaly [10].

In order to explain the depletion of the high-energy neutrino flux in IceCube we assume that the sterile neutrino $\left|v_{4}\right\rangle$ or $\left|v_{5}\right\rangle$ with mass $m_{4}$ or $m_{5}$, and which does not participate in the SBL oscillation, will be almost degenerate in mass with $\left|v_{1}\right\rangle$ or $\left|v_{2}\right\rangle$ and we can estimate its value $\Delta m^{2} \simeq 4.0 \times 10^{-16} \mathrm{eV}^{2}$ in the proceeding section for maximum flavor conversion on Earth. This gives many possibilities for $\Delta m^{2}$ to be considered and we take into account many of them in our analysis as shown in Table 1. A sterile neutrino with a mass of $(1-10) \mathrm{keV}$ is a viable candidate for dark matter [52], can explain the pulsar kicks [53], and can also play a role in other astrophysical phenomena. Finally, to be able to explain the DM problem, we assume that the sixth neutrino mass eigenstate has mass $m_{6} \simeq 1 \mathrm{keV}$ and is almost decoupled from the rest of the neutrinos, both active and sterile.

\section{The mixing matrix}

The matrix $U$ in Eq. (2) is a unitary $6 \times 6$ matrix and in general can be parameterized by 15 real angles and 10 Dirac phases entering directly in the mixing matrix. The remaining five phases enter as a diagonal matrix and sit outside the matrix. The only mixing angles which are measured experimentally are $\theta_{12}, \theta_{23}$, and $\theta_{13}$. In discussing physics beyond the Standard Model scenario one has to incorporate these measured parameters in the analysis pertaining to oscillations involving sterile neutrinos. Models involving one $(3+1)$, two $(3+2)$ and three $(3+3)[43,51,54-60]$ sterile neutrinos have been proposed to explain the discussed discrepancies where many simple parametrization of the matrix $U$ have been used $[56,61]$.

In order to address the aforementioned problems and at the same time accommodate the existing data on the observed oscillations between the active neutrinos we propose the following $6 \times 6$ form for the extended PMNS matrix $U$ :

$U \simeq\left(\begin{array}{ccc|ccc}0.824 & 0.515 & 0.136 & 0.138 & 0.139 & 1.0 \times 10^{-3} \\ -0.501 & 0.527 & 0.583 & 0.243 & 0.203 & 0.174 \\ 0.244 & -0.670 & 0.629 & 0.223 & 0.197 & 0.086 \\ \hline-0.052 & 0.070 & -0.409 & 0.901 & 0.078 & 0.086 \\ -0.050 & -0.047 & -0.261 & -0.214 & 0.935 & 0.085 \\ 0.076 & -0.025 & -0.101 & -0.124 & -0.142 & 0.974\end{array}\right)$.

As discussed in the previous section, we take all the phases to be zero, which makes the $U$ matrix real and after that we vary all the 15 mixing angles, keeping in mind that the $U$ matrix is maintained unitary and with the constraints given by different observations as discussed below. Notice that the first 
$3 \times 3$ block diagonal entries are responsible for explaining the solar, atmospheric, and the reactor neutrino data, and all the mixing matrix elements in this block diagonal are compatible with the constraints given by the experiments. The active $\left(v_{e}, v_{\mu}\right.$, and $\left.v_{\tau}\right)$ content of the three additional mass eigenstates has to be small, which is shown in the first $3 \times 3$ off diagonal block in Eq. (13). Unitarity of $U$ implies the following constraints on the remaining matrix elements:

$X_{i} \equiv \sum_{\alpha=e, \mu, \tau}\left|U_{\alpha i}\right|^{2} \leq 0.3$,

for each $i=4-6$. Similarly for each $\alpha=e, \mu, \tau$

$X_{\alpha} \equiv \sum_{i=4-6}\left|U_{\alpha i}\right|^{2} \leq 0.3$.

Our extended matrix gives $0.04 \leq X_{i} \leq 0.13$ for $i=4-6$ and $0.04 \leq X_{\alpha} \leq 0.13$ for $\alpha=e, \mu, \tau$.

To further tighten the constraint on the active-sterile mixing we can use the effective neutrino mass in $\beta$-decay experiments, which is given by

$m_{e}=\sqrt{\left(\sum_{i}\left|U_{e i}\right|^{2} m_{i}^{2}\right)}$.

This contribution gives the distortion of the electron energy spectrum due to the non-zero neutrino mass and mixing and the current bound on this parameter is $m_{e} \leq 2.2 \mathrm{eV}$ [62]. Similarly in the neutrinoless double beta-decay experiments the effective neutrino mass parameter is given by

$\langle m\rangle_{e e}=\left|\sum_{i} U_{e i}^{2} m_{i}\right|$.

The current bound on this parameter is $\langle m\rangle_{e e}<0.26 \mathrm{eV}$ $[63,64]$. In our analysis, we have three different mass scales; one scale is in the sub-eV range and can even be smaller, making $m_{1}$ degenerate with $m_{2}$. Another scale is of the order of $\mathrm{eV}$, corresponding to either $m_{4}$ or $m_{5}$. The third one is the $\mathrm{keV}$ scale, corresponding to $m_{6}$. The effective neutrino mass parameter in both experiments has to get a contribution mainly from the $\mathrm{keV}$ and $\mathrm{eV}$ mass eigenstates, and to obtain this constraint, we must have $\left|U_{e 6}\right| \leq 10^{-3}$. Our extended $\mathrm{U}$ matrix satisfies this characteristic i.e. $m_{e} \leq 1.05 \mathrm{eV}$ and $\langle m\rangle_{e e} \leq 10^{-3} \mathrm{eV}$. Also, to preserve the well-known mixing between the active neutrinos, the mixing between the active and the sterile neutrinos are required to be small. Thus the flavor mixing matrix elements of the active neutrinos in $U$ (first diagonal block of Eq. 13) constrain the remaining mixing elements between the active and the sterile neutrinos to be small but sizable, translating into the corresponding mixing angles to be a few degrees at most $\left(\theta_{i j} \leq 15^{\circ}\right.$ for $i, j$ from 4 to 6 ).

\section{High-energy astrophysical neutrinos}

It is believed that GRBs which are about $100 \mathrm{Mpc}$ away from us are the sources of UHECRs with energies above $10^{18} \mathrm{eV}$ [17-19]. In the fireball scenario of the GRB emission [20,65], protons are Fermi accelerated to ultra-high energy and constitute probably part of the UHECRs that we observe on Earth. The deep inelastic collision of these high-energy protons with the expanding shock wave as well as with the surrounding background can produce charged and neutral pions. While the decay of a neutral pion can give high-energy gamma rays, the decay of charged pions will produce high-energy neutrinos. So there is some correlation among the UHECRs, high-energy gamma rays, and high-energy neutrinos.

The conventional wisdom is that at the source the flux ratio is $\Phi_{v_{e}}^{0}: \Phi_{v_{\mu}}^{0}: \Phi_{\nu_{\tau}}^{0}=1: 2: 0\left(\Phi_{v_{\alpha}}^{0}\right.$ is the sum of neutrino and anti-neutrino fluxes for the flavor $\alpha$ at the source) due to the decay of charged pions. The vacuum oscillation of these neutrinos on their way to Earth would average to the observed ratio $(1: 1: 1)$ [66]. For high-energy neutrinos above $\sim 1 \mathrm{PeV}$, the muon energy is degraded in a strong magnetic field or gets absorbed in the stellar medium. So high-energy muon neutrinos will be absent and the flux ratio at the source is modified to $(0: 1: 0)$ [67-69]. This will be further modified to $(1: 1.8: 1.8)$ at Earth after vacuum oscillation [70].

Neutron beta decay will also contribute to the neutrino flux ratio. Being neutral, neutrons cannot be accelerated directly by the GRB jet. So these neutrons have to have been produced as secondaries. Around the GRB environment, highenergy neutrons can be produced through the following channels: interaction of Fermi accelerated high-energy protons in the GRB jet with the ambient hydrogen $(p p)$, dissociation of accelerated ions $(A)$ by colliding with the ambient hydrogen $(A p)$, interaction of high-energy protons with the ambient photons $(p \gamma)$, and photodissociation of accelerated ions $(A \gamma)$ [71]. These high-energy secondary neutrons will decay in flight and produce $\bar{v}_{e}$, which will give a flux ratio $(1: 0: 0)$ [67-69]. However, these scenarios have at least one shortcoming: In the GRB environment along with these neutrons, high-energy pions are also produced. The highenergy charged pions will decay to high-energy neutrinos and their energy will be an order of magnitude higher than the $\overline{v_{e}}$ energy produced in neutron beta decay. Also the neutrino flux from pion decay will be higher than the one from the neutron decay. So in an astrophysical environment, a pure neutron source having the flux ratio $(1: 0: 0)$ is highly unrealistic.

The GRB neutrinos travel distances of order $\sim 100 \mathrm{Mpc}$ and neutrino fluxes from these GRBs at different redshifts will be averaged, leading to the averaging of the oscillations. So regardless of their initial flavor content, the flux ratio will be $(1: 1: 1)$, which is one form of decoherence [72]. It 
should also be noted that quantum decoherence will give rise to the same flux ratio [73].

Based on the observed flux of UHECRs, Waxman and Bahcall estimated the neutrino flux, which is $E_{v}^{2} \mathrm{~d} N_{v} / \mathrm{d} E_{v} \sim$ $5 \times 10^{-9} \mathrm{GeVcm}^{-2} \mathrm{~s}^{-1} \mathrm{sr}^{-1}$ in the energy range $\sim 100 \mathrm{TeV}-$ $10 \mathrm{PeV}$ [24]. For GRBs at a redshift of $z \sim 1$ and $L \sim 100$ Mpc with neutrinos energy $100 \mathrm{TeV} \leq E_{v} \leq 10 \mathrm{PeV}$, the maximum flavor conversion will take place for

$4.0 \times 10^{-17} \mathrm{eV}^{2} \leq \Delta m^{2} \leq 4.0 \times 10^{-15} \mathrm{eV}^{2}$.

In other words, the high-energy GRB neutrinos cannot probe a mass squared difference smaller than $\Delta m^{2} \simeq 4.0 \times$ $10^{-17} \mathrm{eV}^{2}$. For our estimate of the neutrino flux we will use this result for the maximum conversion of neutrinos in the IceCube detector. The oscillation length for standard neutrinos as well as for neutrinos satisfying $\Delta m^{2} \sim 1 \mathrm{eV}^{2}$ and $\Delta m^{2} \sim 1 \mathrm{keV}^{2}$ are very short compared to the astrophysical distances, which corresponds to the condition $L \gg L_{\mathrm{osc}}$, and the oscillation probability will be averaged for these cases which will be independent of the neutrino energy and the distance from the source. For our analysis, here we consider the neutrino energy $E_{v}=1 \mathrm{PeV}$, which corresponds to $\Delta m^{2} \simeq 4.0 \times 10^{-16} \mathrm{eV}^{2}$ for maximum flavor conversion on Earth and for this case we replace the oscillatory factor in Eq. (12) by unity.

For the treatment of the neutrino oscillation in Eq. (12), we have neglected the matter effects for GRBs as well as the Earth. The reasons are twofold.

(1) The region of the GRB fireball where these highenergy neutrinos are produced has a very low matter density, which makes the matter potential contribution negligible.

(2) For $\Delta m^{2} \simeq 4.0 \times 10^{-16} \mathrm{eV}^{2}$ the average potential experienced by a $\mathrm{PeV}$ neutrino inside the Earth is $\sqrt{2} G_{F} n_{e} \gg \Delta m^{2} / 2 E_{v}$. Thus, also the Earth's matter has negligible effect on the neutrino oscillation.

\section{Results and discussion}

In the light of insufficient detailed knowledge on the region of the GRB fireball and the region surrounding it where the highenergy neutrinos are produced, as discussed in the previous section, we consider three different flux ratios at the source: the conventional one, $(1: 2: 0)$, the muon-damped source, $(0: 1: 0)$, and the beta beam $(1: 0: 0)$. First of all, it is unclear which flux ratio affects the flux determination on Earth, and secondly there is also uncertainty in the elements of the $U$ matrix and a number of other astrophysical factors: the shape of the neutrino spectra depends on the primary cosmic ray energy spectra and of the target material; and at very high-energy, semileptonic decay of the charm quarks will give rise to extra neutrinos. In our analysis, we neglect the last two uncertainties in calculating the flux ration on Earth.

After traveling a distance $L$, the neutrino flux of a given flavor on Earth is given by

$\Phi_{v_{\alpha}}=\sum_{\beta} P_{\alpha \beta} \Phi_{v_{\beta}}^{0}$

The condition $L \gg L_{\mathrm{osc}}$ is satisfied for the standard neutrinos as well as for neutrinos satisfying $\Delta m^{2} \sim 1 \mathrm{eV}^{2}$ and $\Delta m^{2} \sim$ $1 \mathrm{keV}^{2}$. For all these cases the oscillatory term in Eq. (12) will be replaced by a factor $1 / 2$. For neutrinos traveling a distance beyond $\sim 100 \mathrm{Mpc}$, averaging the sources over the redshift will give an average flux even for very small $\Delta m^{2}$, due to incoherent flavor mixing. We keep the sixth neutrino mass $m_{6}=1 \mathrm{keV}$ fixed throughout the calculation. Table 1 summarizes our findings. We have considered six different possibilities that give a sizable very high-energy neutrino flux depletion in IceCube. In the first four cases we have taken either $m_{4}$ or $m_{5} \sim 1 \mathrm{eV}$. For the remaining cases both $m_{4}$ and $m_{5}$ are almost degenerate with either $m_{1}$ or $m_{2}$, but not both. In these last two cases considered, we do not have $\sim 1 \mathrm{eV}$ neutrino mass and we will be unable to explain the SBL anomaly.

As shown in Table 1, for the initial neutrino flux ratio (1:2:0), we observe that $\Phi_{\nu_{e}}>\Phi_{v_{\mu}}, \Phi_{v_{\tau}}$ is satisfied always. For the mass degeneracy involving $m_{1}$, the electron neutrino flux, $\Phi_{v_{e}}$, is always enhanced from its vacuum value by 6-8\%. At the same time $\Phi_{v_{\mu}}$ decreases by $24-28 \%$ and $\Phi_{\nu_{\tau}}$ by $26 \%$. On the other hand, for the mass degeneracy involving $m_{2}$, while the $\Phi_{v_{e}}$ is decreased by $1-6 \%, \Phi_{v_{\mu}}$ is decreased substantially by $28-38 \%$, and $\Phi_{v_{\tau}}$ is decreased by 11-20\%. The substantial depletion in $\Phi_{v_{\mu}}$ and $\Phi_{\nu_{\tau}}$ in the $(1: 2: 0)$ scenario is due to the sizable mixing of the $v_{\mu}$ and $v_{\tau}$ with the sterile neutrinos, which can be seen from the mixing matrix given in Eq. (13).

Table 1 The first column gives the different possibilities (Pos.) that we have considered. In the second column the mass splittings of the sterile neutrino with the active one are given, where we fix $\Delta m_{i j}^{2} \simeq$ $4.0 \times 10^{-16} \mathrm{eV}^{2}$ for maximum flavor conversion on Earth. The third, the fourth, and the fifth columns give the flux ratio on Earth by considering the flux ratio at the source to be $(1: 2: 0),(0: 1: 0)$, and $(1: 0: 0)$, respectively

\begin{tabular}{lllll}
\hline Pos. & $\Delta m_{i j}^{2}$ & $1: 2: 0$ & $0: 1: 0$ & $1: 0: 0$ \\
\hline I & $\Delta m_{41}^{2}$ & $1.07: 0.75: 0.74$ & $0.28: 0.23: 0.29$ & $0.51: 0.28: 0.16$ \\
II & $\Delta m_{51}^{2}$ & $1.06: 0.76: 0.74$ & $0.28: 0.24: 0.29$ & $0.51: 0.28: 0.16$ \\
III & $\Delta m_{42}^{2}$ & $0.99: 0.69: 0.82$ & $0.23: 0.23: 0.32$ & $0.52: 0.23: 0.19$ \\
IV & $\Delta m_{52}^{2}$ & $0.99: 0.72: 0.80$ & $0.24: 0.24: 0.31$ & $0.52: 0.24: 0.19$ \\
V & $\Delta m_{41,51}^{2}$ & $1.08: 0.72: 0.74$ & $0.30: 0.21: 0.30$ & $0.48: 0.30: 0.14$ \\
VI & $\Delta m_{42,52}^{2}$ & $0.94: 0.62: 0.89$ & $0.22: 0.20: 0.34$ & $0.51: 0.22: 0.21$ \\
\hline
\end{tabular}


For the initial flux ratio $(0: 1: 0)$, as shown in Table 1 , the flux observed on Earth is almost identical for mass degeneracies involving $m_{1}$ (possibilities I and II). Similarly it is also almost identical for degeneracies involving $m_{2}$ (possibilities III and IV). In all these cases we find $\Phi_{v_{\tau}}>\Phi_{v_{\mu}}>\Phi_{v_{e}}$, but we have values still lower than the vacuum oscillation value of $1 / 3$. In the last two cases (possibilities V and VII), the sterile neutrinos with masses $m_{4}$ and $m_{5}$ are taken to be degenerate with either $m_{1}$ or $m_{2}$. We see that the muon neutrino flux is degraded substantially by as much as $38 \%$. In general, our results show that there is a marked decrease in the muon neutrino flux.

For the beta beam flux ratio $(1: 0: 0)$, as shown in the fifth column of Table 1, we observe that $\Phi_{v_{e}}>\Phi_{v_{\mu}}>\Phi_{\nu_{\tau}}$ is always satisfied and in all these cases, while the tau neutrino flux is heavily suppressed (36-43\%), $\Phi_{v_{e}}$ is dramatically increased by as much as $45-58 \%$. The $\Phi_{v_{\mu}}$ is depleted by 9-34 \%. We observe a substantial depletion in muon neutrino flux in the above three scenarios, which may clearly be measurable by IceCube. Without coherence, the depletion in flux due to very small $\Delta m^{2}$ will be smaller.

IceCube can isolate the muon neutrino events from the rest through the track and shower events. The most probable signature of the sterile neutrinos is the depletion of muon neutrino flux due to its mixing with the former. In all the three cases, $(1: 2: 0),(1: 0: 0)$, and $(0: 1: 0)$, we obtain depletion in the muon neutrino flux as well as in the tau neutrino flux. It has also been argued that due to activesterile flavor mixing, there will be an excess of electron neutrinos with a particular energy and zenith angle dependence [74]. For the conventional flux ratio $(1: 2: 0)$ and the beta beam flux ratio $(1: 0: 0)$ we do get an excess of the electron neutrino flux on Earth. In the conventional scenario this excess in $\Phi_{v_{e}}$ is due to the mixing of neutrinos of mass $m_{1}$ with the steriles of mass $m_{4}$ and $m_{5}$, which is absent in the muon-damped scenario $(0: 1: 0)$. But it is to be noted that in the beta beam scenario, the enhancement in $\Phi_{v_{e}}$ can be very high (between 45 and $58 \%$ ) for all activesterile mixing. As argued previously, this scenario is highly unrealistic.

For SBL neutrino oscillations, except for the case of the $\Delta m^{2} \sim 1 \mathrm{eV}^{2}$ term, no other mass square difference will contribute because $\sin ^{2}\left(\pi L / L_{\mathrm{osc}}\right)$ is very small in Eq. (12). In the standard scenario of three active neutrinos, the $v_{\mu}\left(\bar{v}_{\mu}\right) \rightarrow v_{e}\left(\bar{v}_{e}\right)$ oscillation is almost zero, whereas in the present scenario we have a non-zero contribution coming from the mixing of light sterile neutrinos (with mass $m_{4}$ and/or $m_{5}$ ) with active neutrinos. The oscillatory term with $\Delta m_{6 i}^{2} L / 4 E_{v} \gg 1$ for $i=1-5(L \sim 30 \mathrm{~m}$ and $\left.E_{v} \sim 100 \mathrm{MeV}\right)$ will, in principle, contribute to the SBL anomaly. But the mixing of the sixth neutrino is vanishingly small, resulting in a contribution that is negligible. Thus the sixth neutrino decouples from the rest and serves as the non- baryonic dark matter of the Universe $[14,15]$. There are also other explanations for the non-observation of these highenergy neutrinos in IceCube, where it is argued that GRBs may not be the source of high-energy cosmic rays. In that case, there will be no neutrinos. Another explanation is that the GRB fireball calculations of the neutrino flux is subject to sufficiently large astrophysical ambiguities, leading to evading the IceCube limit [29].

Acknowledgments We thank S. Mohanty and S. Pakvasa for valuable comments and discussions. We are also thankful to the anonymous referee for his valuable comments and suggestions. The work of S.S. is partially supported by DGAPA-UNAM (Mexico) Project No. IN103812 and the work of S.R. is partially supported by the Department of Energy (USA) grant No. DE-SC0005366/001.

Open Access This article is distributed under the terms of the Creative Commons Attribution License which permits any use, distribution, and reproduction in any medium, provided the original author(s) and the source are credited.

Funded by $\mathrm{SCOAP}^{3}$ / License Version CC BY 4.0.

\section{References}

1. K. Abe et al., Super-Kamiokande collaboration. Phys. Rev. D 83, 052010 (2011). arXiv:1010.0118 [hep-ex]

2. P. Adamson et al., MINOS collaboration. Phys. Rev. Lett. 106, 181801 (2011). arXiv:1103.0340 [hep-ex]

3. Q. R. Ahmad et al., SNO collaboration. Phys. Rev. Lett. 89, 011301 (2002). [nucl-ex/0204008]

4. S. Abe et al., KamLAND collaboration. Phys. Rev. Lett. 100, 221803 (2008). arXiv:0801.4589 [hep-ex]

5. Y. Fukuda et al., Super-Kamiokande collaboration. Phys. Rev. Lett. 81, 1562 (1998). [hep-ex/9807003]

6. D. G. Michael et al., MINOS collaboration. Phys. Rev. Lett. 97, 191801 (2006). [hep-ex/0607088]

7. Y. Abe et al., DOUBLE-CHOOZ collaboration. Phys. Rev. Lett. 108, 131801 (2012). arXiv:1112.6353 [hep-ex]

8. J. K. Ahn et al., RENO collaboration. Phys. Rev. Lett. 108, 191802 (2012). arXiv:1204.0626 [hep-ex]

9. F. P. An et al., DAYA-BAY collaboration. Phys. Rev. Lett. 108, 171803 (2012). arXiv:1203.1669 [hep-ex]

10. A. Aguilar-Arevalo et al., LSND collaboration. Phys. Rev. D 64, 112007 (2001). [hep-ex/0104049]

11. A. A. Aguilar-Arevalo et al., MiniBooNE collaboration. Phys. Rev. Lett. 105, 181801 (2010). arXiv:1007.1150 [hep-ex]

12. G. Mention, M. Fechner, T. Lasserre, T. A. Mueller, D. Lhuillier, M. Cribier, A. Letourneau, Phys. Rev. D 83, 073006 (2011). arXiv:1101.2755 [hep-ex]

13. S. Schael et al., ALEPH and DELPHI and L3 and OPAL and SLD and LEP Electroweak Working Group and SLD Electroweak Group and SLD Heavy Flavour Group collaborations. Phys. Rep. 427, 257 (2006). [hep-ex/0509008]

14. A. Kusenko, Phys. Rep. 481, 1 (2009). (arXiv:0906.2968 [hep-ph] and references therein)

15. A. Boyarsky, O. Ruchayskiy, M. Shaposhnikov, Ann. Rev. Nucl. Part. Sci. 59, 191 (2009). arXiv:0901.0011 [hep-ph]

16. G.M. Fuller, A. Kusenko, I. Mocioiu, S. Pascoli, Phys. Rev. D 68, 103002 (2003). [astro-ph/0307267]

17. F. Halzen, D. Hooper, Rep. Prog. Phys. 65, 1025 (2002). [astro-ph/0204527] 
18. K. Murase, K. Ioka, S. Nagataki, T. Nakamura, Phys. Rev. D 78, 023005 (2008). arXiv:0801.2861 [astro-ph]

19. J.K. Becker, Phys. Rep. 458, 173 (2008). arXiv:0710.1557 [astro$\mathrm{ph}]$

20. K. Murase, S. Nagataki, Phys. Rev. D 73, 063002 (2006). [astro-ph/0512275]

21. F. Halzen, S.R. Klein, Rev. Sci. Instrum. 81, 081101 (2010). arXiv:1007.1247 [astro-ph.HE]

22. R. Abbasi et al., IceCube collaboration. Nature 484, 351 (2012). arXiv: 1204.4219 [astro-ph.HE]

23. R. Abbasi et al., IceCube collaboration. Phys. Rev. Lett. 106, 141101 (2011). arXiv:1101.1448 [astro-ph.HE]

24. E. Waxman, J.N. Bahcall, Phys. Rev. Lett. 78, 2292 (1997). [astro-ph/9701231]

25. Y. Chikashige, R.N. Mohapatra, R.D. Peccei, Phys. Rev. Lett. 45, $1926(1980)$

26. G.B. Gelmini, M. Roncadelli, Phys. Lett. B 99, 411 (1981)

27. J.F. Beacom, N.F. Bell, D. Hooper, S. Pakvasa, T.J. Weiler, Phys. Rev. Lett. 90, 181301 (2003). [hep-ph/0211305]

28. Z. Li, Phys. Rev. D 85, 027301 (2012). arXiv:1112.2240 [astroph.HE]

29. S. Hummer, P. Baerwald, W. Winter, Phys. Rev. Lett. 108, 231101 (2012). arXiv:1112.1076 [astro-ph.HE]

30. H.-N. He, R.-Y. Liu, X.-Y. Wang, S. Nagataki, K. Murase, Z.-G. Dai, Astrophys. J. 752, 29 (2012). arXiv:1204.0857 [astro-ph.HE]

31. S. Gao, K. Asano, P. Meszaros, JCAP 1211, 058 (2012). arXiv:1210.1186 [astro-ph.HE]

32. P. Baerwald, M. Bustamante, W. Winter, Astrophys. J. 768, 186 (2013). arXiv:1301.6163 [astro-ph.HE]

33. B. Zhang, P. Kumar, Phys. Rev. Lett. 110, 121101 (2013). arXiv:1210.0647 [astro-ph.HE]

34. L. Wolfenstein, Nucl. Phys. B 186, 147 (1981)

35. S.T. Petcov, Phys. Lett. B 110, 245 (1982)

36. S.M. Bilenky, B. Pontecorvo, Sov. J. Nucl. Phys. 38, 248 (1983)

37. S. M. Bilenky, B. Pontecorvo, Lett. Nuovo Cim. 37, 467 (1983)

38. S. M. Bilenky, B. Pontecorvo, Yad. Fiz. 38, 415 (1983)

39. M. Kobayashi, C.S. Lim, Phys. Rev. D 64, 013003 (2001). [hep-ph/0012266]

40. K. E. Cahill, hep-ph/9912416

41. J.F. Beacom, N.F. Bell, D. Hooper, J.G. Learned, S. Pakvasa, T.J. Weiler, Phys. Rev. Lett. 92, 011101 (2004). [hep-ph/0307151]

42. A. Esmaili, Y. Farzan, JCAP 1212, 014 (2012). arXiv:1208.6012 [hep-ph]

43. S. Pakvasa, A. Joshipura, S. Mohanty, Phys. Rev. Lett. 110, 171802 (2013). arXiv:1209.5630 [hep-ph]

44. A. S. Joshipura, S. Mohanty, S. Pakvasa, Phys. Rev. D 89, 033003 (2014). arXiv:1307.5712 [hep-ph]

45. A. Esmaili, F. Halzen, O.L.G. Peres, JCAP 1211, 041 (2012). arXiv:1206.6903 [hep-ph]

46. E. Komatsu et al. WMAP collaboration. Astrophys. J. Suppl. 192, 18 (2011). arXiv:1001.4538 [astro-ph.CO]

47. N. Arkani-Hamed, S. Dimopoulos, S. Kachru, hep-th/0501082

48. R. Mahbubani, L. Senatore, Phys. Rev. D 73, 043510 (2006). [hep-ph/0510064]
49. F. D'Eramo, Phys. Rev. D 76, 083522 (2007). arXiv:0705.4493 [hep-ph]

50. R. Enberg, P.J. Fox, L.J. Hall, A.Y. Papaioannou, M. Papucci, JHEP 0711, 014 (2007). arXiv:0706.0918 [hep-ph]

51. C. Giunti, M. Laveder, Phys. Rev. D 84, 073008 (2011). arXiv:1107.1452 [hep-ph]

52. S. Dodelson, L.M. Widrow, Phys. Rev. Lett. 72, 17 (1994). [hep-ph/9303287]

53. A. Kusenko, G. Segre, Phys. Lett. B 396, 197 (1997). [hep-ph/9701311]

54. M. Archidiacono, N. Fornengo, C. Giunti, A. Melchiorri, Phys. Rev. D 86, 065028 (2012). arXiv:1207.6515 [astro-ph.CO]

55. A. Donini, P. Hernandez, J. Lopez-Pavon, M. Maltoni, T. Schwetz, JHEP 1207, 161 (2012). arXiv:1205.5230 [hep-ph]

56. P. Keranen, J. Maalampi, M. Myyrylainen, J. Riittinen, Phys. Lett. B 574, 162 (2003). [hep-ph/0307041]

57. P.L. Biermann, A. Kusenko, Phys. Rev. Lett. 96, 091301 (2006). [astro-ph/0601004]

58. J. M. Conrad, C. M. Ignarra, G. Karagiorgi, M. H. Shaevitz, J. Spitz, arXiv:1207.4765 [hep-ex]

59. P.S.B. Dev, A. Pilaftsis, Phys. Rev. D 87, 053007 (2013) arXiv: 1212.3808 [hep-ph]

60. A. Merle, Int. J. Mod. Phys. D 22, 1330020 (2013). arXiv: 1302.2625 [hep-ph]

61. V. Gupta, G. Sãanchez-Colõsn, S. Rajpoot, H.-C. Wang, Phys. Rev. D 87, 073009 (2013). arXiv:1304.1065 [hep-ph]

62. C. Kraus, B. Bornschein, L. Bornschein, J. Bonn, B. Flatt, A. Kovalik, B. Ostrick, E. W. Otten et al., Eur. Phys. J. C 40, 447 (2005). [hep-ex/0412056]

63. R. Arnold et al., NEMO collaboration. Phys. Rev. Lett. 95, 182302 (2005). [hep-ex/0507083]

64. M. Bongrand, NEMO-3 collaboration. arXiv:1105.2435 [hep-ex]

65. D.B. Fox, P. Meszaros, New J. Phys. 8, 199 (2006). [astro-ph/0609173]

66. J. G. Learned, S. Pakvasa, Astropart. Phys. 3, 267 (1995). [hep-ph/9408296]

67. J.P. Rachen, P. Meszaros, Phys. Rev. D 58, 123005 (1998). [astro-ph/9802280]

68. P. Lipari, M. Lusignoli, D. Meloni, Phys. Rev. D 75, 123005 (2007). arXiv:0704.0718 [astro-ph]

69. S. Pakvasa, W. Rodejohann, T.J. Weiler, JHEP 0802, 005 (2008). arXiv:0711.4517 [hep-ph]

70. T. Kashti, E. Waxman, Phys. Rev. Lett. 95, 181101 (2005). [astro-ph/0507599]

71. R.M. Crocker, M. Fatuzzo, R. Jokipii, F. Melia, R.R. Volkas, Astrophys. J. 622, 892 (2005). [astro-ph/0408183]

72. D.J. Wagner, T.J. Weiler, Mod. Phys. Lett. A 12, 2497 (1997). arXiv:1101.5677 [astro-ph.CO]

73. D. Hooper, D. Morgan, E. Winstanley, Phys. Lett. B 609, 206 (2005). [hep-ph/0410094]

74. F. Halzen, J. Phys. Conf. Ser. 408, 012023 (2013). arXiv: 1111.0918 [hep-ph] 